\title{
Uso de tomografia computadorizada no diagnóstico e planejamento endodôntico de pré-molar superior com dupla curvatura radicular
}

\author{
Use of computed tomography in endodontic diagnosis and planning of maxillary premolar with
} double radicular curvature

Uso de tomografía computarizada en el diagnóstico y planificación endodóntica de premolar superior con doble curvatura radicular

Recebido: 15/09/2021 | Revisado: 19/09/2021 | Aceito: 26/09/2021 | Publicado: 27/09/2021

\author{
Bruno Soares Machado \\ ORCID: https://orcid.org/0000-0002-0961-758X \\ Universidade Cruzeiro do Sul, Brasil \\ E-mail: bsmachado91@hotmail.com \\ André Hayato Saguchi \\ ORCID: https://orcid.org/0000-0002-1903-4986 \\ Universidade Cruzeiro do Sul, Brasil \\ E-mail: andre_saguchi@yahoo.com.br \\ Ângela Toshie Araki Yamamoto \\ ORCID: https://orcid.org/0000-0003-4402-7531 \\ Universidade Cruzeiro do Sul, Brasil \\ E-mail: angela.araki@cruzeirodosul.edu.br \\ Michele Baffi Diniz \\ ORCID: https://orcid.org/0000-0002-0693-2162 \\ Universidade Cruzeiro do Sul, Brasil \\ E-mail: mibdiniz@hotmail.com
}

\begin{abstract}
Resumo
Introdução: Os exames de imagem auxiliam no correto diagnóstico e planejamento do tratamento endodôntico. A tomografia computadorizada de feixe cônico ou cone beam (TCCB) é um recurso auxiliar na Endodontia, sendo favorável ao tratamento comparada à radiografia periapical por permitir avaliar tecidos duros da região maxilofacial e estruturas tridimensionais em casos complexos, além da possibilidade de visualização corte a corte. Objetivo: Relatar um caso clínico de um paciente com necessidade de tratamento endodôntico em pré-molar superior com curvatura radicular, sendo a tomografia computadorizada de feixe cônico empregada como exame complementar para avaliação da anatomia radicular, diagnóstico e planejamento. Relato do caso: Paciente do sexo feminino, 37 anos, apresenta dente fraturado com dor pulsante, contínua e localizada há 6 meses. Ao exame radiográfico periapical digital e confirmação por TCCB observou-se dilaceração radicular no terço médio do dente 15 , com dupla curvatura e lesão periapical extensa, com envolvimento do dente 14. O diagnóstico foi de periodontite apical crônica nos dentes 14 e 15 . Após 6 meses de acompanhamento radiográfico e por TCCB, observou-se redução da lesão periapical de ambos os dentes submetidos a tratamento endodôntico, aspecto de normalidade das características clínicas e ausência de sintomatologia dolorosa. Conclusão: A TCCB foi de extrema importância para o correto diagnóstico, planejamento e sucesso do tratamento endodôntico de segundo pré-molar superior com dupla curvatura radicular, devendo ser indicada na fase de avaliação anatômica do paciente, uma vez que pode estar associada às falhas na localização, instrumentação e obturação dos canais radiculares, comprometendo o resultado do tratamento.
\end{abstract}

Palavras-chave: Tomografia Computadorizada Cone Beam; Endodontia; Terapia endodôntica.

\begin{abstract}
Introduction: Imaging exams help in the correct diagnosis and planning of endodontic treatment. Cone beam or cone beam computed tomography (CBCT) is an auxiliary resource in Endodontics, being favorable to the treatment compared to periapical radiography because it allows the evaluation of hard tissues of the maxillofacial region and threedimensional structures in complex cases, in addition to the possibility of view cut by cut. Objective: To report a clinical case of a patient in need of endodontic treatment in a maxillary premolar with root curvature, being the cone beam computed tomography used as a complementary exam for the evaluation of the root anatomy, diagnosis and planning. Case report: A 37-year-old female patient presents with a fractured tooth with continuous, localized pulsating pain for 6 months. Digital periapical radiographic examination and confirmation by CBCT revealed root dilaceration in the middle third of tooth 15 , with double curvature and extensive periapical lesion involving tooth 14 . The diagnosis was chronic apical periodontitis in teeth 14 and 15. After 6 months of radiographic and CBCT follow-up, a reduction in the
\end{abstract}


periapical lesion of both teeth submitted to endodontic treatment was observed, with normal clinical characteristics and absence of painful symptoms. Conclusion: CBCT was extremely important for the correct diagnosis, planning and success of endodontic treatment of maxillary second premolars with double root curvature, and should be indicated in the anatomical assessment phase of the patient, as it may be associated with failures in the location, instrumentation and filling of the root canals, compromising the result of the treatment.

Keywords: Cone Beam Computed Tomography; Endodontics; Endodontic therapy.

\section{Resumen}

Introducción: Los exámenes por imágenes ayudan en el diagnóstico y la planificación correctos del tratamiento de endodoncia. La tomografía computarizada de haz cónico o de haz cónico (TCCB) es un recurso auxiliar en Endodoncia, siendo favorable al tratamiento en comparación con la radiografía periapical porque permite la evaluación de tejidos duros de la región maxilofacial y estructuras tridimensionales en casos complejos, además de posibilidad de vista corte a corte. Objetivo: Presentar un caso clínico de un paciente con necesidad de tratamiento endodóntico en un premolar superior con curvatura radicular, utilizándose la TCCB como examen complementario para la evaluación de la anatomía radicular, diagnóstico y planificación. Caso clínico: Una paciente de 37 años se presenta con un diente fracturado con dolor pulsátil localizado continuo durante 6 meses. El examen radiográfico periapical digital y la confirmación por TCCB reveló desgarro radicular en el tercio medio del diente 15, con doble curvatura y lesión periapical extensa, afectando al diente 14. El diagnóstico fue periodontitis apical crónica en los dientes 14 y 15 . Después de 6 meses de radiografía y TCCB. En el seguimiento se observó una reducción de la lesión periapical de ambos dientes sometidos a tratamiento endodóntico, con características clínicas normales y ausencia de sintomatología dolorosa. Conclusión: La TCCB fue de suma importancia para el correcto diagnóstico, planificación y éxito del tratamiento endodóntico de los segundos premolares superiores con doble curvatura radicular, debiendo estar indicada en la fase de valoración anatómica del paciente, ya que puede estar asociada a fallas en la localización, instrumentación y relleno de los conductos radiculares, comprometiendo el resultado del tratamiento.

Palabras clave: Tomografía Computarizada Cone Beam; Endodoncia; Terapia endodóntica.

\section{Introdução}

Na prática endodôntica, os exames de imagem se fazem muito importantes frente ao correto diagnóstico e planejamento de tratamento (Lopez et al., 2014). Durante muito tempo, a radiografia periapical foi o exame de imagem mais recomendado em tratamento endodônticos, no entanto, apresenta como limitação a visualização bidimensional, muitas vezes sobrepondo imagens, gerando resultados falso-positivos e falso-negativos no diagnóstico da doença e estruturas vitais ao tratamento (Koc, Sonmez, Yilmaz, Karahan, \& Kamburoglu, 2018).

O sucesso no tratamento endodôntico está relacionado ao diagnóstico, quantidade de paredes instrumentadas e obturação em toda a extensão dos canais radiculares. Dessa forma, o profissional deve estar familiarizado com as possíveis variações dos sistemas de canais radiculares. Sendo assim, exames de imagem podem revelar dificuldades anatômicas, patologias persistentes ou secundárias e fracassos em tratamentos prévios (Baruwa et al., 2020; do Carmo et al., 2021; Hou, 2018; Keine et al., 2015). Estudos transversais com radiografias mostram alta prevalência de periodontite apical associada a dentes com tratamento endodôntico (22-61\%) e de falhas no preenchimento do canal cirúrgico (47\%-86\%)(Kirkevang, Horsted-Bindslev, Orstavik, \& Wenzel, 2001; Kirkevang, Orstavik, Horsted-Bindslev, \& Wenzel, 2000; Odesjo, Hellden, Salonen, \& Langeland, 1990).

Nesse sentido, a tomografia computadorizada de feixe cônico ou cone beam (TCCB) tem sido muito utilizada como recurso auxiliar na Endodontia, sendo favorável ao tratamento comparada à radiografia periapical por permitir avaliar tecidos duros da região maxilofacial e estruturas tridimensionais em casos complexos, além da possibilidade de visualização corte a corte, possibilitando uma avaliação precisa e melhor planejamento do tratamento pelo profissional (Alswilem, Abouonq, Iqbal, Alajlan, \& Alam, 2018; Matherne, Angelopoulos, Kulild, \& Tira, 2008).

Portanto, o objetivo deste trabalho foi relatar um caso clínico de adulto jovem com necessidade de tratamento endodôntico no qual a TCCB foi empregada como exame complementar de extrema importância para avaliação da anatomia radicular, diagnóstico e correto planejamento. 


\section{Caso clínico}

Paciente do sexo feminino, 37 anos de idade, leucoderma, procurou atendimento odontológico em uma Clínica de Especialidade de Endodontia, queixando-se de um dente fraturado, com dor pulsante, contínua e localizada, com duração da sintomatologia por cerca de 6 meses. Durante a anamnese, não foram relatadas alterações sistêmicas e de saúde geral por parte da paciente. A paciente assinou um Termo de Consentimento Livre e Esclarecido, autorizando a documentação e divulgação do caso.

Ao exame clínico, observou-se restauração provisória inadequada com fratura coronária nas faces distal, palatina e oclusal do dente 15, além de gengivite (Figura 1). A paciente apresentou resposta negativa ao teste térmico, positiva ao teste de percussão horizontal e negativa ao teste de percussão vertical. Ao exame radiográfico periapical digital observou-se dilaceração radicular no terço médio do dente 15, com dupla curvatura e lesão periapical extensa, onde aparentemente envolvia também o dente 14 (Figura 2). Com o possível envolvimento do dente 14, foram realizados o teste térmico, com resposta negativa, e testes de percussão vertical e horizontal negativas.

Figura 1. (a) Aspecto clínico inicial.

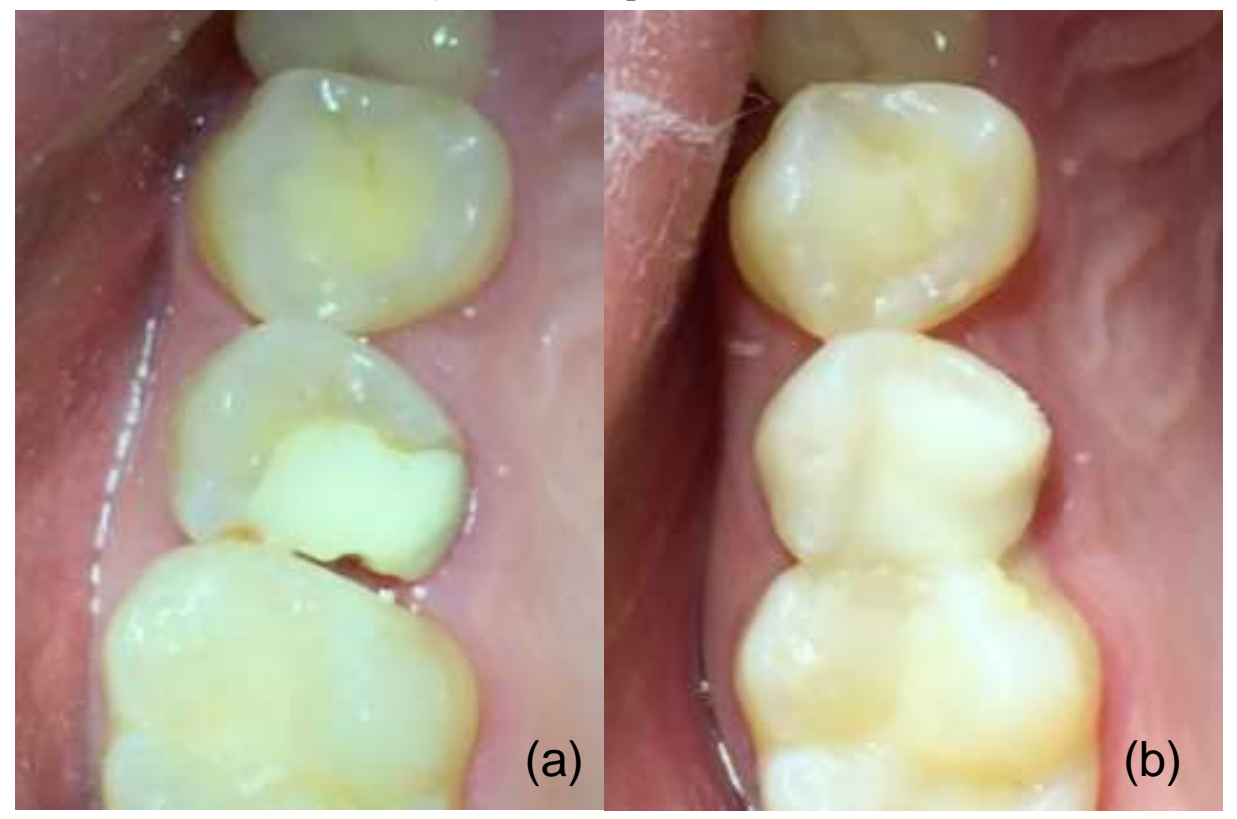

Notar restauração inadequada com fratura no dente 15. (b) Imagem final do dente 14 após restauração em resina composta. Fonte: Autores.

Figura 2. Radiografias inicial e final.

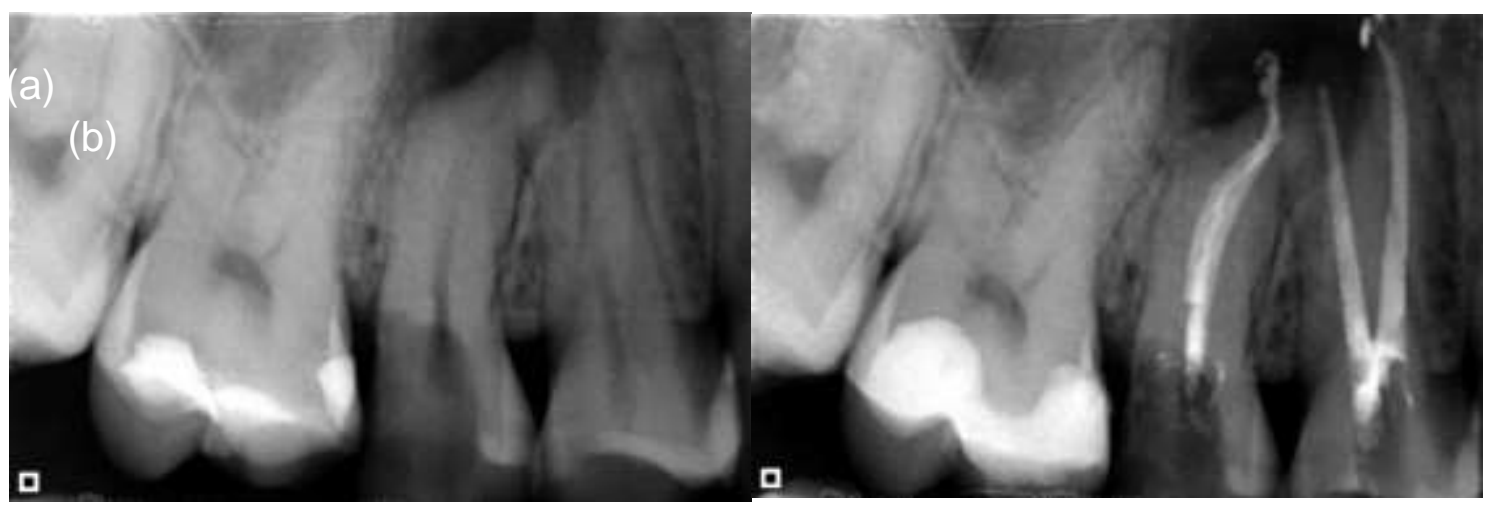

Radiografia Inicial imediata demonstrando a curvatura acentuada no dente 15 e lesão periapical aparentemente envolvendo o dente 14 e dente 15. (b) imagem final pós tratamento endodôntico e completo preenchimento de ambos os dentes. Fonte: Autores. 
Perante a situação clínica e radiográfica da paciente, foi solicitado um exame complementar de TCCB para avaliação anatômica e trajeto radicular do dente 15 . Verificou-se então a presença de um único conduto, com a confirmação da dupla curvatura na região média e apical, além do envolvimento da lesão periapical no dente 14 (Figura 3). Com base nos achados, o diagnóstico foi periodontite apical crônica nos dentes 14 e 15. Assim, a paciente foi submetida ao tratamento endodôntico em dupla sessão.

Figura 3. Imagem tomográfica pré e pós tratamento.

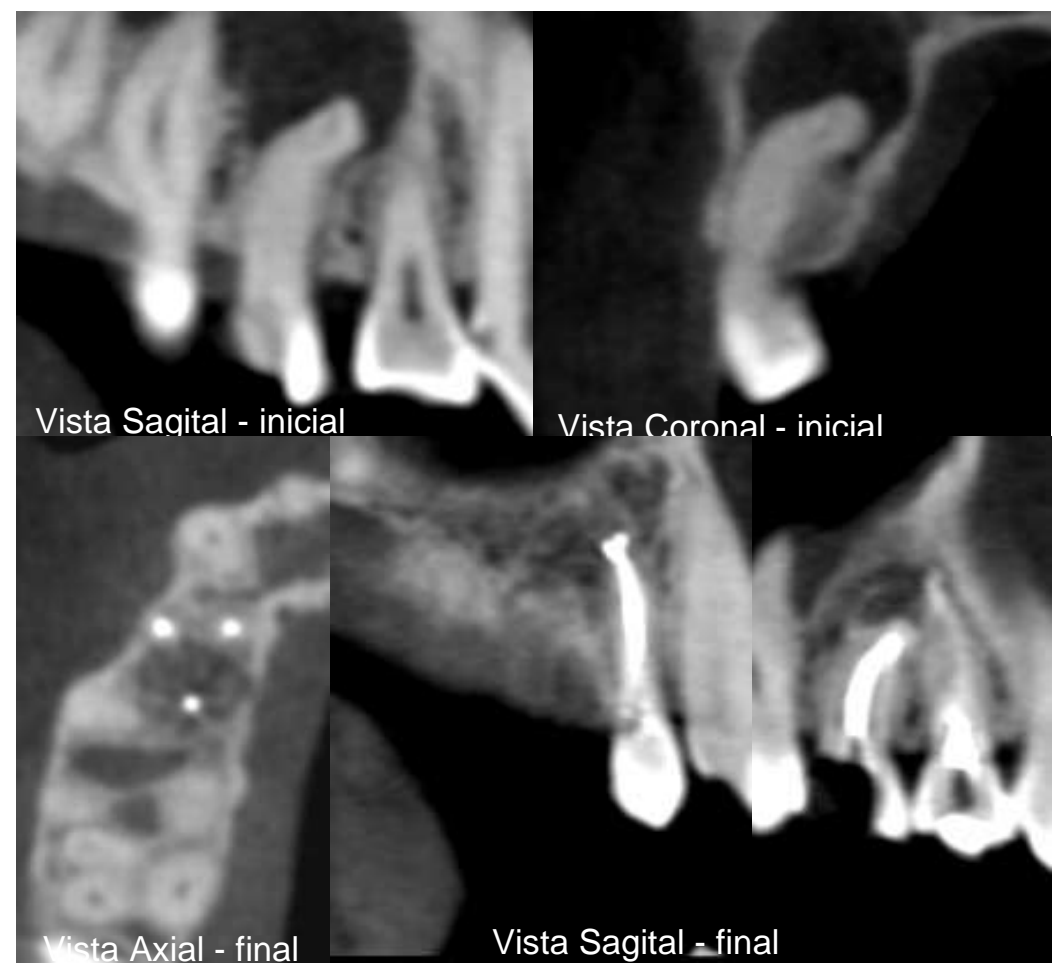

Observa-se a dupla curvatura radicular do dente 15 (distal e palatina). Fonte: Autores.

Na primeira sessão, foi realizada anestesia infiltrativa com lidocaína Alphacaine 2\% 1:100.000 (Nova DFL, Rio de Janeiro - RJ, Brasil), seguido de isolamento absoluto do campo operatório com lençol em látex (Madeitex Indústria e Comércio de Artefatos de Latex Ltda, São José dos Campos - SP, Brasil), cirurgia de acesso dos dentes 14 e 15 com broca esférica número 1012 HL até a trepanação da câmara pulpar e remoção do teto com broca endo Z-K (JET, São Paulo - SP, Brasil), irrigação com hipoclorito de sódio a 2,5\% (Asfer, São Caetano do Sul - SP, Brasil), penetração desinfetante do terço cervical e médio com limas do tipo K (Dentsply Maillefer, Ballaigues, Suiça). Após a penetração desinfetante concluída foi realizado o préalargamento do terço cervical e médio com lima R25 (VDW-dental, GmbH, Munique, Alemanha) calibrada em 17mm, correspondente a $3 \mathrm{~mm}$ aquém do comprimento aparente do dente, com movimentos de bicada conforme recomendação do fabricante. A odontometria foi realizada com localizador apical digital Romiapex A-15 (Romidan Ltd, Giv'at Shmu'el, Israel), e estabelecido os devidos comprimentos de trabalho para cara conduto radicular e realizado o preparo químico-cirúrgico a $1 \mathrm{~mm}$ aquém do ápice após confirmação radiográfica do comprimento de trabalho. O preparo químico-cirúrgico foi realizada com limas em níquel-titânio R25 e o preparo apical com a lima R40. Foi realizada a irrigação de $20 \mathrm{ml}$ de hipoclorito de sódio 2,5\% e ativação por 15 segundos com ponta ultrassônica Irrisonic (Helso, Santa Rosa de Viterbo - SP, Brasil) e realizada a irrigação de 20 ml EDTA-T (Fórmula e Ação Farmácia, São Paulo - SP, Brasil) também com ativação ultrassônica por 15 segundos e nova ativação do hipoclorito de sódio 2,5\% por 15 segundos. Os canais foram secos com cânulas de aspiração Capillary Tip 0,014mm (Ultradent do Brasil Produtos Odontológicos, Indaiatuba - SP, Brasil) e realizada a medicação intra-canal com hidróxido de 
cálcio Ultracal XS (Ultradent do Brasil Produtos Odontológicos, Indaiatuba - SP, Brasil) e selamento provisório com cimento de ionômero de vidro Vidrion R (S.S. White Duflex, Rio de Janeiro - RJ, Brasil). O retorno da paciente foi agendado após 14 dias.

Na segunda sessão, foi realizada a reinstrumentação com lima R40, irrigação abundante com hipoclorito de sódio a 2,5\% e ativação por 15 segundos, irrigação de $20 \mathrm{ml}$ de EDTA-T com ativação ultrassônica por 15 segundos e nova irrigação com hipoclorito de sódio 2,5\%. A obturação foi realizada a partir da técnica de cone único com cone de guta percha (Tanariman Industrial LTDA, Amazonas, Brasil) calibrada em ponta 40 e conicidade 0,06 . Ao término da sessão, foi realizada radiografia periapical final, na qual foi possível observar o preenchimento completo dos canais dos dentes 14 e 15 (Figura 2) e selamento provisório dos dentes com cimento de ionômero de vidro.

Em uma terceira sessão, foi realizada a restauração final na face oclusal do dente 14 com resina composta Z350 (3M/ESPE, Sumaré-SP, Brasil) (Figura 1). Para o dente 15, a paciente foi encaminhada para reabilitação protética, no entanto, ainda não o fez por motivos alheios. A paciente foi orientada da necessidade da reabilitação para garantir uma boa reparação óssea e prognóstico do caso.

A paciente foi orientada a retornos periódicos a cada 3 meses para avaliação clínica e radiográfica para acompanhamento do tratamento realizado. Após 6 meses, observou-se radiograficamente e na TCCB (Figura 3) a redução da lesão periapical de ambos os dentes submetidos a tratamento endodôntico, aspecto de normalidade das características clínicas e ausência de sintomatologia dolorosa.

\section{Discussão}

O presente caso descreve a importância da TCCB para avaliação, diagnóstico e planejamento endodôntico de um segundo pré-molar superior com curvatura radicular. De acordo com Burklein et al. (2017), a anatomia radicular interna e externa de pré-molares superiores varia de acordo com a raça e etnia, e as essas variações anatômicas desafiam o clínico durante o tratamento endodôntico (Burklein, Heck, \& Schafer, 2017).

A variabilidade de raízes em pré-molares superiores está intimamente ligada ao achatamento mésio-distal durante seu processo de formação (rizogênese), podendo apresentar multiplicidade de raízes, e consequentemente, multiplicidade de condutos radiculares onde somente 11\% dos pré-molares superiores (Cleghorn, Christie, \& Dong, 2007). Além disso, o elemento dental pode sofrer também alterações de forma radicular, como curvaturas, duplas curvaturas e dilacerações radiculares, no entanto, apenas 1,7\% dos pré-molares superiores apresentam dilaceração radicular ("Dilaceration among Nigerians: prevalence, distribution, and its relationship with trauma - Udoye - 2009 - Dental Traumatology - Wiley Online Library," 2018). Assim, essas situações clínicas geram dificuldade de diagnóstico e planejamento de tratamento endodôntico (Ozyurek, Yilmaz, \& Uslu, 2017).

De maneira geral, o diagnóstico e planejamento endodôntico é realizado com auxílio de radiografias periapicais, com base na sua imagem bidimensional. No entanto, a TCCB deve ser indicada como exame de imagem complementar não-invasivo, acurado, que permite a avaliação dentária e seus tecidos adjacentes de forma tridimensional, em alta resolução, visando explorar a anatomia radicular e auxiliar no planejamento endodôntico (Michetti, Maret, Mallet, \& Diemer, 2010; Mota de Almeida, Knutsson, \& Flygare, 2014; Patel, Dawood, Wilson, Horner, \& Mannocci, 2009; Patel et al., 2015; Pauwels et al., 2012). No presente caso clínico, notou-se ao exame radiográfico periapical curvatura radicular do segundo pré-molar superior direito, necessitando de exame de imagem complementar por meio da TCCB para um correto diagnóstico da dupla curvatura do dente 15 e plano de tratamento, reduzindo significativamente o tempo de trabalho durante o tratamento endodôntico.

Além disso, a TCCB foi importante para escolha do instrumento endodôntico adequado empregado no caso apresentado, por conta da dupla curvatura radicular do elemento dental 15 e alta flexibilidade dos instrumentos Reciproc, que possuem corpo 
metálico em formato de dupla vírgula em seu corte transversal e tratamento térmico aplicado no instrumento. Assim, a seleção do instrumento é uma etapa endodôntica primordial para o bom prognóstico do caso, pois ele pode interferir na qualidade de preparo e capacidade de evitar desvios do conduto principal(Cleghorn et al., 2007).

Com base na avaliação tomográfica, foi observada a presença de achatamentos, istmos e regiões de intercomunicação entre os condutos do dente 15, apresentando maior dificuldade para o correta sanificação. Portanto, foi utilizado hipoclorito de sódio a 2,5\% com ativação ultrassônica na fase de irrigação final, conforme proposto por Mozo et al. (2014), visto que em casos de complexidade anatômica do elemento dental e, consequentemente do canal radicular, a fase de descontaminação por meio de irrigantes se faz ainda mais importante devido a diminuição do contato do instrumento endodôntico com a parede dentinária, gerando menor desgaste dentinário e menor descontaminação (Bürklein, Hinschitza, Dammaschke, \& Schäfer, 2012; Das, De Ida, Nair, Saha, \& Chattopadhyay, 2017; Ozyurek et al., 2017). Assim, a literatura sugere o aumento da concentração do irrigante na intenção de se obter ação bactericida sobre o remanescente orgânico no interior do canal radicular (Mohammadi, 2008; Violich \& Chandler, 2010).

Deve-se ressaltar que a TCCB permite a avaliação da lesão periapical em tamanho real, sua extensão, natureza e localização, além de fraturas radiculares, anatomia radicular e topografia do osso alveolar (Patel et al., 2009). No entanto, apresenta algumas limitações, como doses de radiação e custo mais elevado comparado às radiografias periapicais e panorâmicas, necessidade de encaminhamento do paciente ao local especializado para sua a aquisição, limitações dos algoritmos usados na formação da imagem adquirida, geração de artefatos indesejáveis por objetivos metálicos, necessidade do paciente ficar completamente imóvel durante a aquisição da imagem tomográfica, além de expertise do profissional para correta interpretação do caso (Patel et al., 2009; Patel et al., 2015). Assim, o profissional deve se basear tanto na filosofia do princípio ALARA (As Low As Reasonably Achievable), ou seja, "tão baixo quanto possível”, como ALADA (As Low As Diagnostically Acceptable), ou seja, "tão baixo quanto aceitável" (Jaju \& Jaju, 2015) para diagnóstico do uso das radiações ionizantes, devendo ser considerado somente quando técnicas radiográficas convencionais não são suficientes para o correto diagnóstico endodôntico (Patel et al., 2009; Patel et al., 2015).

\section{Considerações Finais}

De acordo com o caso clínico apresentado, conclui-se que a TCCB foi de extrema importância como exame de imagem complementar para o correto diagnóstico, planejamento e sucesso do tratamento endodôntico de segundo pré-molar superior com dupla curvatura radicular, devendo ser indicada na fase de avaliação anatômica do paciente, uma vez que pode estar associada às falhas na localização, instrumentação e obturação dos canais radiculares, comprometendo o resultado do tratamento.

\section{Agradecimentos}

Agradecemos à $\operatorname{Prof}^{\mathrm{a}} \operatorname{Dr}^{\mathrm{a}}$ Ângela Toshie Araki pela colaboração e apoio durante o processo de elaboração deste trabalho.

\section{Referências}

Alswilem, R., Abouonq, A., Iqbal, A., Alajlan, S. S., \& Alam, M. K. (2018). Three-Dimensional Cone-Beam Computed Tomography Assessment of Additional Canals of Permanent first Molars: A Pinocchio for Successful Root Canal Treatment. J Int Soc Prev Community Dent, 8(3), 259-263. 10.4103/jispcd.JISPCD_3_18

Baruwa, A. O., Martins, J. N. R., Meirinhos, J., Pereira, B., Gouveia, J., Quaresma, S. A., \& Ginjeira, A. (2020). The Influence of Missed Canals on the Prevalence of Periapical Lesions in Endodontically Treated Teeth: A Cross-sectional Study. J Endod, 46(1), 34-39 e31. 10.1016/j.joen.2019.10.007

Burklein, S., Heck, R., \& Schafer, E. (2017). Evaluation of the Root Canal Anatomy of Maxillary and Mandibular Premolars in a Selected German Population Using Cone-beam Computed Tomographic Data. J Endod, 43(9), 1448-1452. 10.1016/j.joen.2017.03.044

Bürklein, S., Hinschitza, K., Dammaschke, T., \& Schäfer, E. (2012). Shaping ability and cleaning effectiveness of two single-file systems in severely curved root canals of extracted teeth: Reciproc and WaveOne versus Mtwo and ProTaper. Int Endod J, 45(5), 449-461. 10.1111/j.1365-2591.2011.01996.x 
Cleghorn, B. M., Christie, W. H., \& Dong, C. C. (2007). The root and root canal morphology of the human mandibular first premolar: a literature review. $J$ Endod, 33(5), 509-516. 10.1016/j.joen.2006.12.004

Das, S., De Ida, A., Nair, V., Saha, N., \& Chattopadhyay, S. (2017). Comparative evaluation of three different rotary instrumentation systems for removal of gutta-percha from root canal during endodontic retreatment: An in vitro study. J Conserv Dent, 20(5), 311-316. 10.4103/jcd.jcd_132_17

Dilaceration among Nigerians: prevalence, distribution, and its relationship with trauma - Udoye - 2009 - Dental Traumatology - Wiley Online Library. (2018). 10.1111/j.1600-9657.2009.00796.x

do Carmo, W. D., Verner, F. S., Aguiar, L. M., Visconti, M. A., Ferreira, M. D., Lacerda, Mfls, \& Junqueira, R. B. (2021). Missed canals in endodontically treated maxillary molars of a Brazilian subpopulation: prevalence and association with periapical lesion using cone-beam computed tomography. Clin Oral Investig, 25(4), 2317-2323. 10.1007/s00784-020-03554-4

Hou, B. X. (2018). [Role of the operating microscope in diagnosis and treatment of endodontic diseases]. Zhonghua Kou Qiang Yi Xue Za Zhi, 53(6), 386-391. 10.3760/cma.j.issn.1002-0098.2018.06.005

Jaju, P. P., \& Jaju, S. P. (2015). Cone-beam computed tomography: Time to move from ALARA to ALADA. Imaging Sci Dent, 45(4), 263-265. 10.5624/isd.2015.45.4.263

Keine, K. C., Kuga, M. C., Pereira, K. F., Diniz, A. C., Tonetto, M. R., Galoza, M. O., \& de Andrade, M. F. (2015). Differential Diagnosis and Treatment Proposal for Acute Endodontic Infection. J Contemp Dent Pract, 16(12), 977-983.

Kirkevang, L. L., Horsted-Bindslev, P., Orstavik, D., \& Wenzel, A. (2001). Frequency and distribution of endodontically treated teeth and apical periodontitis in an urban Danish population. Int Endod J, 34(3), 198-205.

Kirkevang, L. L., Orstavik, D., Horsted-Bindslev, P., \& Wenzel, A. (2000). Periapical status and quality of root fillings and coronal restorations in a Danish population. Int Endod J, 33(6), 509-515.

Koc, C., Sonmez, G., Yilmaz, F., Karahan, S., \& Kamburoglu, K. (2018). Comparison of the accuracy of periapical radiography with CBCT taken at 3 different voxel sizes in detecting simulated endodontic complications: an ex vivo study. Dentomaxillofac Radiol, 47(4), 20170399. 10.1259/dmfr.20170399

Lopez, F. U., Kopper, P. M., Cucco, C., Della Bona, A., de Figueiredo, J. A., \& Vier-Pelisser, F. V. (2014). Accuracy of cone-beam computed tomography and periapical radiography in apical periodontitis diagnosis. J Endod, 40(12), 2057-2060. 10.1016/j.joen.2014.09.003

Matherne, R. P., Angelopoulos, C., Kulild, J. C., \& Tira, D. (2008). Use of cone-beam computed tomography to identify root canal systems in vitro. J Endod, 34(1), 87-89. 10.1016/j.joen.2007.10.016

Michetti, J., Maret, D., Mallet, J. P., \& Diemer, F. (2010). Validation of cone beam computed tomography as a tool to explore root canal anatomy. J Endod, 36(7), 1187-1190. 10.1016/j.joen.2010.03.029

Mohammadi, Z. (2008). Sodium hypochlorite in endodontics: an update review. Int Dent J, 58(6), 329-341.

Mota de Almeida, F. J., Knutsson, K., \& Flygare, L. (2014). The effect of cone beam CT (CBCT) on therapeutic decision-making in endodontics. Dentomaxillofac Radiol, 43(4). 10.1259/dmfr.20130137

Odesjo, B., Hellden, L., Salonen, L., \& Langeland, K. (1990). Prevalence of previous endodontic treatment, technical standard and occurrence of periapical lesions in a randomly selected adult, general population. Endod Dent Traumatol, 6(6), 265-272.

Ozyurek, T., Yilmaz, K., \& Uslu, G. (2017). Shaping Ability of Reciproc, WaveOne GOLD, and HyFlex EDM Single-file Systems in Simulated S-shaped Canals. J Endod, 43(5), 805-809. 10.1016/j.joen.2016.12.010

Patel, S., Dawood, A., Wilson, R., Horner, K., \& Mannocci, F. (2009). The detection and management of root resorption lesions using intraoral radiography and cone beam computed tomography - an in vivo investigation. Int Endod J, 42(9), 831-838. 10.1111/j.1365-2591.2009.01592.x

Patel, S., Durack, C., Abella, F., Shemesh, H., Roig, M., \& Lemberg, K. (2015). Cone beam computed tomography in Endodontics - a review. Int Endod J, 48(1), 3-15. 10.1111/iej.12270

Pauwels, R., Beinsberger, J., Collaert, B., Theodorakou, C., Rogers, J., Walker, A., \& Horner, K. (2012). Effective dose range for dental cone beam computed tomography scanners. Eur J Radiol, 81(2), 267-271. 10.1016/j.ejrad.2010.11.028

Violich, D. R., \& Chandler, N. P. (2010). The smear layer in endodontics - a review. Int Endod J, 43(1), 2-15. 10.1111/j.1365-2591.2009.01627.x 